\title{
Pseudomembranous Conjunctivitis
}

National Cancer Institute

\section{Source}

National Cancer Institute. Pseudomembranous Conjunctivitis. NCI Thesaurus. Code C35196.

Conjunctivitis that is characterized by formation of a pseudomembrane. 